\title{
GLEE, a new computer program for glass electrode calibration
}

\author{
Peter Gans ${ }^{\mathrm{a}, *}$, Brendon O’Sullivan ${ }^{\mathrm{b}}$ \\ a School of Chemistry, The University of Leeds, Leeds LS2 9JT, UK \\ ${ }^{\mathrm{b}}$ Latimer Hall, College of Chemistry, University of California at Berkeley, Berkeley, CA 94720-1460, USA
}

Received 25 March 1999; received in revised form 28 June 1999; accepted 3 August 1999

\begin{abstract}
A new computer program (GLEE, glass electrode evaluation) has been written for the calibration of a glass electrode by means of a strong acid-strong base titration. This program provides an estimate of the carbonate contamination of the base, the pseudo-Nernstian standard potential and slope of the electrode and, optionally, the concentration of the base and $\mathrm{p} K_{\mathrm{W}}$. The program runs under the Windows $3.1 \mathrm{x}, 9 \mathrm{x}$ and NT operating systems. (C) 2000 Elsevier Science B.V. All rights reserved.
\end{abstract}

Keywords: Glass electrode calibration; Potentiometry; Stability constants

The calibration of glass electrodes in terms of hydrogen ion concentration may be performed following IUPAC recommendations [1] which are based on the use of either one or two designated buffer solutions, or on the basis of a regression procedure performed with data from more than two buffer solutions [2,3]. These procedures are not wholly satisfactory for potentiometric titrations using a background medium of high ionic strength, as is usual in the determination of stability constants. An alternative procedure is to perform a titration of strong acid with strong base as a source of solutions with known hydrogen ion concentration [4]. The method seems to have been

\footnotetext{
* Corresponding author. Tel.: + 44-113-2336409; fax: + 44113-2336565.

E-mail address: peterg@chem.leeds.ac.uk (P. Gans)
}

first published by McBryde [5] and has been the subject of detailed statistical analysis [6,7]. An interesting aspect of the use of MAGEC [7] was the way in which it and MINIQUAD [8] were used in a cyclic process.

The program GLEE has been developed as part of the Hyperquad suite of programs for stability constant determination [9]. It was written in Visual Basic and runs under the Windows $3.1 \mathrm{x}$, 95/98 and NT operating systems on a personal computer. It uses a (non-linear) least-squares refinement to fit a modified Nernst Eq. (1),

$E=E^{0}+s \lg \left[H^{+}\right]$

where $E$ is a measured electrode potential, $E^{0}$ and $s$ are parameters of the refinement and represent the standard electrode potential and slope and 
$\left[\mathrm{H}^{+}\right]$represents the hydrogen ion concentration. $E^{0}$ and $s$ are used in Hyperquad data input. Since stability constant determinations are usually carried out in a medium of high ionic strength Eq. (1) is an excellent approximation apart from in the regions of high acidity or basicity where junction potentials may be not negligible.

In acid solutions the hydrogen ion concentration is obtained from the mineral acid concentration, $T_{\mathrm{H}}$, as calculated from Eq. (2), that is, $\lg \left[H^{+}\right]=\lg \left(T_{\mathrm{H}}\right)$.

$T_{\mathrm{H}}=\frac{a_{\mathrm{H}} v_{0}+\gamma b_{\mathrm{H}} v}{v_{0}+v_{1}+v}$

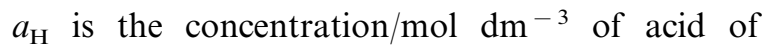
which $v_{0} \mathrm{~cm}^{3}$ were added to the titration vessel, $b_{\mathrm{H}}$ is the concentration $/ \mathrm{mol} \mathrm{dm}{ }^{-3}$ of base in the burette (by convention given a negative sign), $v_{1}$ is the volume $/ \mathrm{cm}^{3}$ of background electrolyte solution added to the titration vessel, and $v / \mathrm{cm}^{3}$ is the volume of base added from the burette. $\gamma$ is a correction factor for the base concentration: if $\gamma$ is refined the calculated base concentration is $\gamma b_{\mathrm{H}}$.

In alkaline solutions the effective concentration of the base is usually reduced by the presence of a small amount (preferably $<1 \%$ ) of carbonate contamination. The extent of this contamination

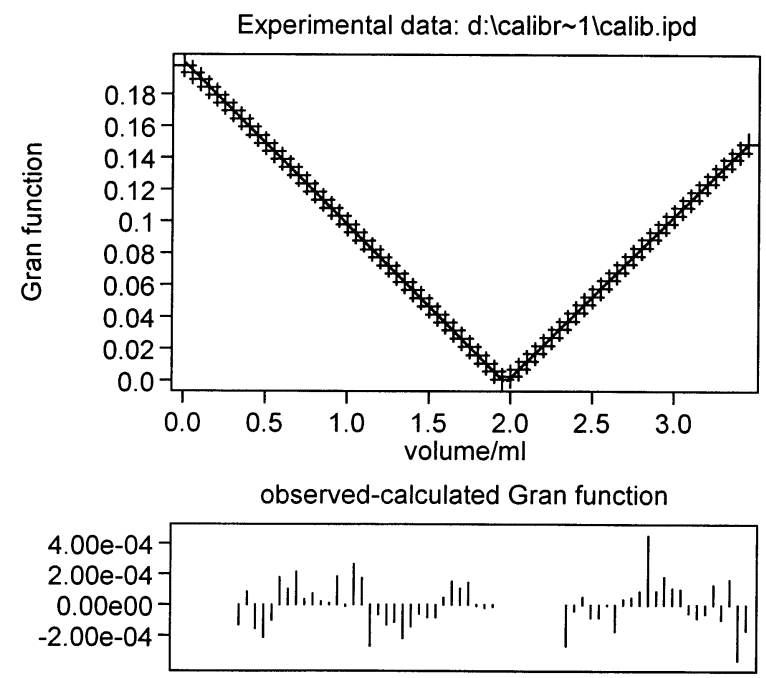

Fig. 1. GLEE output showing the Gran plot. All the experimental data points are shown, but residuals are only shown for those points that were used for the calculation. can be estimated by means of a Gran plot [10]. Initially $E^{0}$ is estimated from the acid region [11] and $s$ is taken as the ideal Nernstian slope $(T /$ $5.0399 \mathrm{mV}$ ). Then the functions (3) and (4) are fitted by linear least-squares.

Acid region:

$\left(v_{0}+v_{1}+v\right) 10^{\frac{E-E^{0}}{s}}=m^{a} v+c^{a}$

Alkaline region:

$$
\left(v_{0}+v_{1}+v\right) 10^{\frac{E^{0}-E}{s}-p K_{\mathrm{w}}}=m^{b} v+c^{b}
$$

A typical Gran plot is shown in Fig. 1. From the slopes and intercepts of the fitted lines two estimates are obtained of the volume of base consumed at the equivalence point: $v_{e}^{a}=-c^{a} / m^{a}$ from the acid region and $v_{e}^{b}=-c^{b} / m^{b}$ from the alkaline region. Assuming that the difference is due to carbonate the effective base concentration is reduced by the factor $v_{e}^{a} / v_{e}^{b}$ in the alkaline region. The mineral acid concentration in the alkaline region is then given by Eq. (5).

$T_{H}=\frac{a_{H} v_{0}+\gamma \frac{v_{e}^{a}}{v_{e}^{b}} b_{H} v}{v_{0}+v_{1}+v}$

$T_{\mathrm{H}}$ is negative and $\lg \left[\mathrm{H}^{+}\right]=-p K_{\mathrm{W}}-\lg \left(-T_{\mathrm{H}}\right)$.

With these estimates of $\lg \left[\mathrm{H}^{+}\right]$the standard potential and slope can be obtained by leastsquares fitting of Eq. (1). The whole process, including the Gran plot is repeated with the refined values of $E^{0}$ and $s$. An example of the graphical output is shown in Figs. 2 and 3. Numerical output is also shown on the computer screen and may be sent to a printer.

Since both $\gamma$ and $p K_{\mathrm{w}}$ are used in calculating $\lg \left[\mathrm{H}^{+}\right]$these quantities may also be fitted by the least-squares process. To accommodate all possibilities a non-linear refinement algorithm is used. However, the refinement of $p K_{\mathrm{w}}$ is strongly discouraged; the help file associated with the program contains extensive tabulations of values of $p K_{\mathrm{w}}$ which have been reported in the literature [12-14]. The data used for the calculation are obtained from a file of volume, e.m.f. data pairs, but individual data points may be included or excluded from the calculations. 

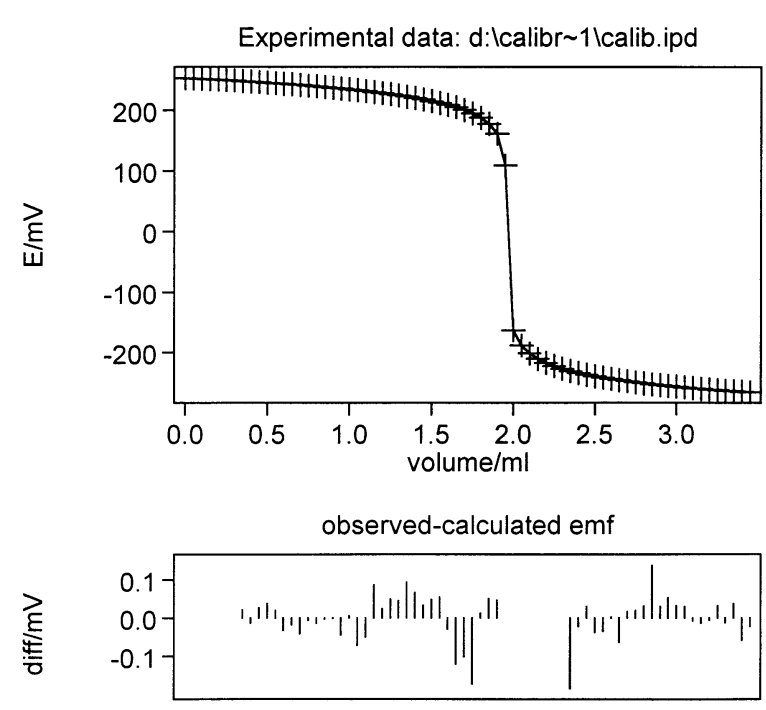

Fig. 2. GLEE output showing measured electrode potentials and residuals as a function of titre volume. Residuals are only shown for those data points that were used for the calculation.

Figs. 1-3 were produced directly by GLEE, copied to the clipboard as Windows Metafiles and pasted into Word to be submitted for publication without retouching. In all three figures points between $\mathrm{pH} 9.5$ and 10.6 have been excluded from the calculation as this is the region where some bicarbonate ion is present in equilibrium with

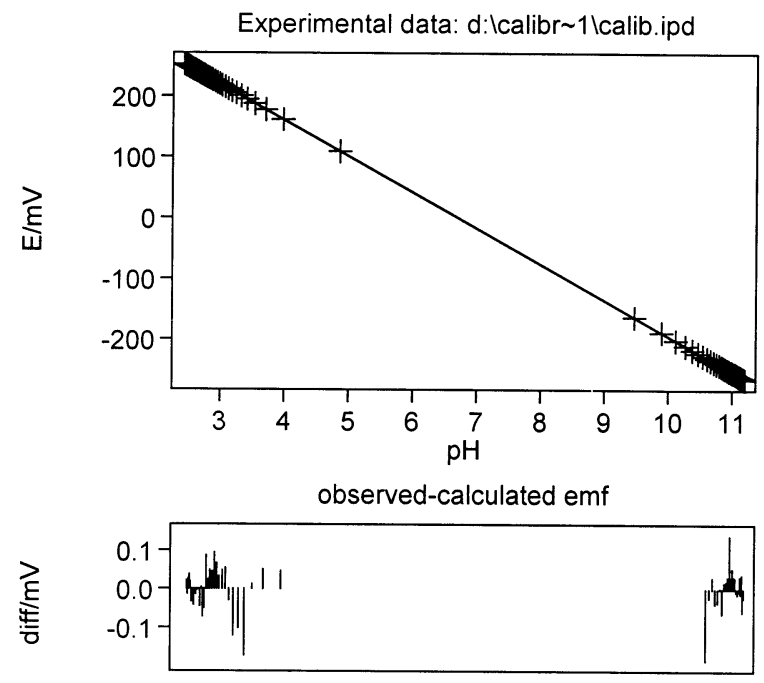

Fig. 3. GLEE output showing measured electrode potentials and residuals as a function of calculated $-\lg \left[\mathrm{H}^{+}\right]$. carbonate $\left(\mathrm{HCO}_{3}^{-} \rightleftharpoons \mathrm{CO}_{3}^{2-}+\mathrm{H}^{+}, \quad p K_{a} \approx 9.9\right)$. The program GLEE may be downloaded free of charge from http://chem.leeds.ac.uk/People/ Peter_Gans/glee.htm

\section{Experimental}

All solutions were made with ' $\mathrm{CO}_{2}$ free' water. For this milliQ water (resistivity $>18 \mathrm{M} \Omega \mathrm{cm}$ ) was boiled and cooled under a stream of nitrogen. Electrolyte solution $(0.1 \mathrm{M} \mathrm{KCl})$ was prepared by dissolution of A.C.S. grade $\mathrm{KCl}$ (Fisher). Standard acid $(\mathrm{HCl})$ and base $(\mathrm{KOH})$ solutions $(\approx 0.1$ M) were prepared by dilution of Baker Dilut-it ampoules. All solutions were kept in their storage vessels for $24 \mathrm{~h}$ before use; the base solution was stored under a small positive pressure of ascaritescrubbed argon gas.

The base solution was standardised against a weighed amount of potassium hydrogen phthalate. The acid solution was standardised against borax. Phenolphthalein and Congo red were used as indicators for end-point determination. Each solution was re-standardised until its concentration was known to within $0.2 \%$ (typically requiring six replications). The titrations were conducted manually using the same apparatus as for the potentiometric titrations. This approach eliminated any variation that may be caused by the autoburette.

The titration cell was built 'in-house' and composed of glass with a teflon lid. The lid had various openings for insertion of the electrode, burette tip and gas inlet and outlet. The cell was held under a positive pressure of argon to eliminate the ingress of $\mathrm{CO}_{2}$ and thermostatted at $25 \pm 0.1^{\circ} \mathrm{C}$.

A Metrohm autoburette (Titrino 702 SM) was used for titrant delivery and for the collection of electrode potential readings. This instrument was controlled via a serial (RS-232C) interface to a personal computer. Data collection was automated using modules written in the $G$ language of the LABVIEW [15] programming environment. The glass bulb electrode used was a Corning high performance reference combination electrode (catalogue no. 476146). 
Titrations for electrode calibration were performed by combining $50.0 \mathrm{~cm}^{3}$ of electrolyte (from an A-grade pipette) with $2.000 \mathrm{~cm}^{3}$ of standardised $\mathrm{HCl}$ solution (from the autoburette) in the titration cell. Metrohm autoburettes allow convenient switching of the titrant by the use of 'exchange units'. Thus the standardised base solution was introduced and the titration performed by the addition of $0.05 \mathrm{~cm}^{3}$ aliquots. $30 \mathrm{~s}$ were allowed after each addition of titrant for equilibration. After that, electrode potential readings were collected at $3 \mathrm{~s}$ intervals until nine successive measurements agreed to within $\pm 0.1 \mathrm{mV}$. Typically this was achieved within 9-15 measurements except in the poorly buffered region near the endpoint ( $\mathrm{pH} 4.5-9.5)$. At each point the mean of the final 9 measurements was recorded.

\section{Discussion}

Accurate calibration of the electrode is crucial to the determination of stability constants by potentiometric titrations, as any error in the calibration becomes a systematic error in the interpretation of titration data. However the process is far from easy because of the interdependence of all the parameters involved. The strong acid/ strong alkali method is convenient, but its accuracy depends on the use of primary standards to establish the concentrations of the acid and alkali. We have used potassium hydrogen phthalate to standardise the alkali and borax for the acid. An alternative would be to use constant-boiling-point hydrochloric acid as the primary standard [16]. Another feature of this method is that the electrode is calibrated directly in terms of hydrogen ion concentration, as is needed for the calculation of a concentration quotient, although this scale is not unique [17].

An important feature of GLEE is that allowance is made for the seemingly inevitable contamination of the base by carbonate - the equivalence point derived from the Gran plot in the alkaline region was always found to be larger than that obtained from the acid region. The use of Eq. (5) results in a much better fit of the experimental data than when a single base con- centration is used for both acid and alkaline regions. Indeed, the excellent agreement between observed and calculated electrode potentials, illustrated in Fig. 2, provides good evidence for the validity of Eqs. (1) and (5).

The presence of carbonate in the base should be regarded as a systematic error to be reduced as much as possible by good laboratory practice. Nevertheless, by taking carbonate contamination into account GLEE is able to fit the experimental calibration curve to within experimental error over the $\mathrm{pH}$ ranges $2.5-4.5$ and $10.7-11.5$ (in the vicinity of the end-point electrode readings are relatively unreliable). Outside this range the effects of varying junction potentials and/or alkaline error become apparent. This range of linear electrode response is generally adequate for stability constant computation by means of a program such as Hyperquad [9].

When a stock solution of alkali was repeatedly standardised over a period of weeks it was observed that the carbonate contamination level apparently rose slowly but steadily. Since the apparatus was maintained under a positive pressure of argon ingress of carbon dioxide seemed unlikely. An alternative possibility is that the alkali is attacking its glass containing vessel with dissolution of silicate which mimics the behaviour of carbonate to some extent.

\section{References}

[1] A.K. Covington, R.G. Bates, R.A. Durst, Pure Appl. Chem. 57 (1985) 531.

[2] A. Avdeef, J.J. Bucher, Anal. Chem. 50 (1978) 2137.

[3] F.G.K. Baucke, R. Naumann, C. Alexander-Weber, J. Am. Chem. Soc. 65 (1993) 3244.

[4] R.J. Motekaitis, A.E. Martell, The Determination and Use of Stability Constants, VCH, New York, 1988.

[5] W.A.E. McBryde, Analyst 94 (1969) 337.

[6] A. Braibanti, C. Bruschi, E. Fisicaro, M. Pasquali, Talanta 33 (1986) 471.

[7] P.M. May, D.R. Williams, in: D.J. Leggett (Ed.), Computational Methods For The Determination of Formation Constants, Plenum Press, New York, 1985, p. 37.

[8] A. Sabatini, A. Vacca, P. Gans, Talanta 21 (1974) 53.

[9] P. Gans, A. Sabatini, A. Vacca, Talanta 43 (1996) 1739.

[10] G. Gran, Analyst 77 (1952) 661.

[11] F.J.C. Rossotti, H. Rossotti, J. Chem. Educ. 42 (1965) 375 . 
[12] I. Kron, S.L. Marshall, P.M. May, G. Hefter, E. Königsberger, Monatsh. 126 (1995) 819.

[13] F.H. Sweeton, R.E Mesmer, C.F. Baes, J. Solution Chem. 3 (1974) 191.

[14] H.S Harned, B.B. Owen, The Physical Chemistry of Electrolytic Solutions, third ed., Reinhold Publishing,
New York, 1958.

[15] http://www.natinst.com/.

[16] A.I. Vogel, A Textbook of Quantitative Inorganic Analysis, Longman, London, 1978.

[17] H. Sigel, A.D. Zuberbühler, O. Yamauchi, Anal. Chim. Acta 255 (1991) 63. 